\title{
Congenital Urogenital Tract Anomalies are Associated with a Higher Rate of Radiologic Interpretation Error
}

\author{
Johnson J L' ${ }^{1 *}$, Mirasol S1, Kapila $C^{1}$, Ok T ${ }^{1}$ and \\ Siddighi $\mathrm{S}^{2}$ \\ ${ }^{1}$ Loma Linda University School of Medicine, Loma Linda \\ University, USA \\ ${ }^{2}$ Division of Female Pelvic Medicine Reconstructive \\ Surgery, Loma Linda University, USA \\ *Corresponding author: J ohnson J L, Loma Linda \\ University School of Medicine, 11175 Campus St, Loma \\ Linda, CA 92350, USA
}

Received: August 04, 2021; Accepted: September 03, 2021; Published: September 10, 2021

\begin{abstract}
Objective: Congenital Urogenital Tract Anomalies (CUTA) are complex and proper diagnosis is important for competent patient care. We set out to investigate whether there is an increased rate of radiologic image interpretation error in patients with CUTA.

Methods: We utilized a case-control study design to compare each CUTA case $(n=30)$ with 6 age-matched controls $(n=180)$. We evaluated electronic medical records obtained from EPIC of patients who were referred to our tertiary care center for a higher level of care. We compared imaging results from MRI, $\mathrm{CT}$, and US to intraoperative findings to look for any discrepancies in four key anatomical areas (vagina, uterus, ovaries, and urologic-kidney/ureter/bladder). Error was determined using a standardized scoring system for adjudicating imaging discrepancies in each anatomic area.
\end{abstract}

Results: Cases and controls were similar across all demographic variables except for weight, (controls had a higher BMI). The rate of radiologic interpretation error was higher in patients with congenital urogenital tract anomalies when compared to age-matched controls (OR=51.65; 95\% Cl $(6.39,417.42)$ ).

Conclusion: CUTA are associated with a high rate of radiologic interpretation error. Although radiologic scans are helpful in the work up of CUTA, imaging results may not be definitively diagnostic.

Keywords: Congenital urogenital anomalies; Urogenital tract anomalies; Radiologist interpretation error; Mayer-Rokitansky-Küster-Hauser; Vaginal agenesis

\section{Abbreviations}

CUTA: Congenital Urogenital Tract Anomalies; MRKH: MayerRokitansky-Küster-Hauser; CT: Computer Tomography; MRI: Magnetic Resonance Imaging; US: Ultrasound; LLUH: Loma Linda University Health; BMI: Body Mass Index; OR: Odds Ratio; CI: Confidence Interval; KUB: Kidney, Ureter, and Bladder

\section{Introduction}

While most women are born with typical female anatomy, woman can rarely be born with congenital urogenital tract anomalies (CUTA) of the reproductive tract. Incidence of uterine anomalies has been estimated at $5.5 \%$ in the general population [1]. These anomalies can range from disorders of the vulva (e.g., congenital adrenal hyperplasia), disorders of the vagina (e.g., imperforate hymen or vaginal septa), and disorders of the uterus such as complete absence, rudimentary horns, or the rarest, cervical agenesis $[2,3]$. Since both genital and urinary systems in females arise from a similar embryonic origin, called Müllerian ducts, there is a high association between genital tract abnormalities and urological anomalies [4]. In fact, one study found for patients with didelphic uteri, $70 \%$ had an absent kidney [5]. These anatomic differences may not be discovered until patients are in puberty, and they even may be missed by healthcare providers during work-up. Obtaining accurate diagnosis through radiologic imaging is important not only in counseling the patient and her family about future fertility and sexual potential, but also in guiding gynecology surgeons during reparative surgery.

There may be a higher incidence of radiologic interpretation error in patients with congenital reproductive tract anomalies, especially in patients with Müllerian agenesis (or MRKH) [2]. While patients with congenital anomalies may not be directly injured because of radiologic image misinterpretation, surgeons still rely heavily on radiologists' interpretation of images to make decisions about patient management as well as prognosis before the operation. Thus, accurate image interpretation is essential for competent patient care. In addition, being incorrectly diagnosed with a congenital anomaly brings psychological complications and uncertainty for young patients when it comes to their future reproductive and sexual potential.

One study found a $26 \%$ inter-observer discrepancy rate and a $32 \%$ intra-observer discrepancy rate for 90 abdominal and pelvic CTs without any congenital anomalies reviewed by three radiologists that specialized in abdominal imaging [6]. While error has been evaluated in common radiological examinations, CT radiological error for congenital urogenital anomalies has not been thoroughly studied. Typically, CT has no place in diagnosis of female genital anomalies [7]. Different variations of uteri can be distinguished via ultrasound, and it is a non-invasive, simple and low-cost part of women's routine evaluation [7]. Ultrasound interpretation error has not been studied extensively in congenital anomalies, even though it is used for initial 
evaluation in most cases. Preibsch et al. found that in patients with $\mathrm{MRKH}$, uterine rudiments on magnetic resonance imaging (MRI) disagreed with laparoscopy $21.8 \%$ of the time [8].

In this study, we attempt to broadly quantify radiologic interpretation error in patients with congenital urogenital tract anomalies compared to a control group. Our null hypothesis is that the rate of radiologic interpretation error is similar between congenital anomaly cases and age-matched controls.

\section{Materials and Methods}

This case control study was approved by the Institutional Review Board at Loma Linda University Health (IRB\# 5160217) (LLUH). The medical records of patients undergoing gynecological surgery from 2009 to 2020 at a single institution were reviewed from EPIC electronic medical records. Cases were selected from the surgical records of patients with reproductive tract anomalies who were referred to our tertiary care center for a higher level of care due to complexity. All patients were included who were diagnosed with a congenital reproductive tract anomaly requiring surgery and who also had preoperative imaging with Magnetic Resonance Imaging (MRI), Computed Tomography (CT), and/or Ultrasound (US). Congenital anomalies included in this study consisted of disorders of the vulva, vagina, cervix, and uterus as well as any associated urologic anomalies (Table 1). Cases were excluded that had a noncongenital reproductive tract anomaly diagnosis, and/or did not have preoperative imaging on file.

For each case, approximately 6 controls were selected who were age-matched to approximately $+/-5$ years and who were also referred to our institution for higher level of care. Controls were selected from all patients in our institution's gynecology and gynecology/oncology services who underwent any type of surgery where the status of the reproductive tract could be assessed and who also had preoperative radiologic studies (MRI, CT, and/or US) during the years 2009-2020.

We collected data about relevant independent variables such as age, race, BMI, and medical insurance to ensure that the control group was as similar to the cases as possible. Distance of each patient's hometown from our institution for subjects and controls also was collected to ensure that the control group did not consist of local patients admitted through the emergency department and were in fact referred to LLUH for higher level of care due to complexity. We also collected information about the radiologist who interpreted the images as well as the imaging center to assess for other confounding variables.

For each patient, MRI, CT and/or ultrasound imaging interpretations for 4 key anatomical areas (vagina, uterus, ovaries, and urologic consisting of kidneys/ureters/bladder) were compared with the surgical findings in the operating room for the same four areas, with the surgical findings as the gold standard. Each anatomic area for a given imaging study was given a score ranging from $0-3$, with 0 representing no discrepancies between imaging and operative findings, 1 representing slight discrepancy, 2 representing clinically significant discrepancy, and 3 representing major discrepancies between imaging and operative findings. For instance, an MRI finding of a short uterine length compared with a surgical finding of a normal uterus would be assigned a score of 1 , while a surgical finding of didelphys compared with an MRI detecting no didelphys would be assigned a score of 2 . A score of 3 would be assigned if the anatomical area detected on imaging was totally absent upon surgery or vice versa: for instance, if a normal uterus was present but the imaging findings indicated absence of uterus, that interpreted image of the uterus would be assigned score of 3 . The imaging study (MRI, $\mathrm{CT}$, or ultrasound) was considered to be in error if the score across all anatomic areas added up to $>2$ points. This type of scoring was performed in a standard fashion for both cases as well as controls. Four evaluators each independently assigned the aforementioned points, and any differences in scoring were settled by discussion and a majority vote between all 4 raters. Inter-rater reliability was assessed using intraclass correlation coefficient and Cronbach's Alpha, and both were high, 0.772 and 0.788 , respectively. Correlation coefficient analysis was performed using IBM SPSS Statistics for Windows (Version 27.0. Armonk, NY: IBM Corp).

Continuous variables (BMI, Age, and distance of patient's hometown to LLU) were analyzed using the Wilcoxson two sample test. Proportions, such as race and insurance, were analyzed using the chi-squared test. A two-sided alpha of less than 0.05 was considered statistically significant. Summary Statistical analyses were performed in SAS software (version 9.4; SAS Institute Inc., Cary, NC, USA). We included every congenital anomaly patient from our service since 2009 due to a low number of eligible subjects; therefore, power analysis was not done prior to collecting data. Instead, post-hoc power analysis was performed after data collection and was high (99\%).

\section{Results}

In this study, 1,850 patient charts were screened for eligibility between the years 2009 and 2020. Of these, 1,820 patients were excluded leaving 30 patients who met the inclusion criteria. A variety of congenital anomalies were examined with MRKH, transverse septum and uterine didelphys being the most common anomalies (Table 2). Each case patient in this study was age-matched with 6 controls totaling 180 controls. The cases were similar to controls on all demographic variables except the controls had a higher BMI. As seen in Table 3, the rate of radiologic interpretation error was higher in patients with congenital urogenital tract anomalies versus age-

Table 1: Congenital urogenital tract anomalies (CUTA).

\begin{tabular}{|l|c|}
\hline \multicolumn{1}{|c|}{ Congenital Anomaly } & Quantity \\
\hline MRKH & $20 \%$ \\
\hline Uterine didelphys & $15 \%$ \\
\hline Transverse vaginal septum & $13 \%$ \\
\hline Hymenal abnormalities (imperforate, cribriform) & $10 \%$ \\
\hline Longitudinal vaginal septum & $8 \%$ \\
\hline Renal agenesis & $8 \%$ \\
\hline Vaginal stenosis/atresia (congenital) & $5 \%$ \\
\hline Cervical agenesis & $5 \%$ \\
\hline CAH (Congenital Adrenal Hyperplasia) & $5 \%$ \\
\hline Bicornuate uterus & $5 \%$ \\
\hline VACTERL syndrome & $2 \%$ \\
\hline Androgen Insensitivity Syndrome & $2 \%$ \\
\hline Gartner's duct cyst & $2 \%$ \\
\hline
\end{tabular}


Table 2: Demographics, baseline characteristics.

\begin{tabular}{|c|c|c|c|}
\hline & Study Group $(n=30)$ & Control $(n=180)$ & P-values \\
\hline \multicolumn{4}{|l|}{ Age } \\
\hline 10-32 (SD) & $18.3(5.0)$ & $19.9(4.9)$ & 0.068 \\
\hline BMI & $24.9(8.2)$ & $28.6(8.1)$ & 0.003 \\
\hline $\begin{array}{l}\text { Distance from LLU } \\
\text { (miles) }\end{array}$ & $33.2(39.4)$ & $26.5(49.1)$ & 0.186 \\
\hline \multicolumn{4}{|l|}{ Race } \\
\hline White & 19 & 67 & \multirow{5}{*}{0.069} \\
\hline Hispanic & 6 & 81 & \\
\hline Black & 3 & 18 & \\
\hline Asian & 1 & 5 & \\
\hline Other & 1 & 9 & \\
\hline \multicolumn{4}{|l|}{ Insurance } \\
\hline Government & 18 & 92 & \multirow{4}{*}{0.764} \\
\hline Private & 11 & 77 & \\
\hline None & 1 & 9 & \\
\hline Other & 0 & 2 & \\
\hline
\end{tabular}

matched controls $(\mathrm{OR}=51.65 ; 95 \% \mathrm{CI}(6.39,417.42))$. There was no pattern of radiologic interpretation error across radiologist or center where imaging was performed, and thus these were not significant variables.

\section{Discussion}

In this study we have demonstrated that radiologic interpretation error in patients with congenital urogenital anomalies is significantly higher than our control group ( $\mathrm{OR}=52)$.

The search for limits of diagnostic imaging modalities is not new. One study found that MRI imaging is helpful and sometimes preferred due to its non-invasiveness, but it is still "unclear" if it can be used as a diagnostic tool for pelvic fractures [9]. Another study revealed that CT scans have an inter-and intra-reader discrepancy rate of $32 \%$ and $26 \%$, respectively [6]. Preibsch et al. found that although the accuracy of MRI on MRKH patients could be as high as $97 \%$ for ovaries, it drops to as low as $78 \%$ for uterine rudiments [8]. They found that MRI underestimated small uterine rudiments in $41.8 \%$ of cases, which reveals a limitation of MRI; furthermore, the sensitivity of ultrasound in detecting rudiments is even lower. In addition, MRI did not fully agree with the laparoscopic localization of ovaries in $22 \%$ of patients [8].

Although MRI, CT, and US are helpful in the work-up of patients with congenital urogenital tract anomalies, they are not definitively diagnostic. It is important for the ordering physician to communicate with the radiologist regarding the working-diagnosis of the patient along with information about what they are looking for on imaging. This may guide the radiologist to improve interpretation. The result of this research suggests that diagnostic laparoscopy may be important to definitively diagnose congenital urogenital tract abnormalities. However, considering the small number of cases and wide confidence interval in our study, the question of whether only MRKH, or all congenital urogenital abnormalities need laparoscopy still needs to be answered. We would also recommend that patients with complex urogenital tract anomalies be referred to physicians and/or centers with experience in managing these patients.

There are several reasons for a higher rate of radiologic interpretation error. Firstly, these anomalies are complicated due to numerous possibilities in anatomic variation, which can occur from embryologic error. In addition, these anomalies are often associated with endometriosis, which can cause adhesions and distortion of anatomy making interpretation more difficult. Also, since congenital anomalies are rare, radiologists may have less experience interpreting these images. If there are questionable findings on imaging, radiologists may be more inclined to default to "normal." Finally, all imaging modalities are associated with artifacts, which may make interpretation more difficult.

There are several limitations to this study. We chose a casecontrol study design because congenital urogenital tract anomalies are rare. Because this study is a retrospective case-control study, information bias may have confounded the results. Additionally, there is a possibility that relevant data was missing. For example, some radiography reports did not have any observation about one organ system in question. In this situation, however, we gave the radiologist the benefit of the doubt and scored the organ as normal. Congenital urogenital tract anomalies are rare, and only 30 patients met our criteria for being cases in this study [1]. Thus, we had to combine all the imaging results (i.e. MRI, CT, and US) as well as all congenital reproductive tract anomalies (Table 1) of subjects in order to increase the power of our study. We did the same for the control group. Thus, we cannot make any conclusions about interpretation error of MRI versus US, since all imaging studies were lumped into one category. The small sample size resulted in a wide confidence interval in our estimate of the odds ratio $95 \%$ CI $[6.39,417.42]$. The case-control comparison shows that the control group is significantly heavier (higher BMI), which could have confounded the results. However, Voss et al. demonstrated that more radiographic errors were found among obese patients; therefore, we do not believe this BMI difference negatively affected our results.

Table 3: Error points assigned representing discrepancies between imaging and operative findings.

Table 3: Error points assigned representing discrepancies between imaging and operative findings.
\begin{tabular}{|l|c|c|c|c|c|}
\hline \multirow{2}{*}{} & \multicolumn{2}{|c|}{ MRI } & \multicolumn{2}{c|}{ CT } & US \\
\cline { 2 - 6 } & Cases $(\mathbf{n = 2 2})$ & Control (n=36) & Cases (n=8) & Control (n=67) & Cases (n=25) \\
\hline Vagina & 2 & 0 & 1 & 0 & 0 \\
\hline Uterus & 1 & 0 & 0 & 0 & 0 \\
\hline Ovary & 1 & 0 & 0 & 0 & 0 \\
\hline KUB & 0 & 0 & 2 & 0 & 0 \\
\hline Total Error & 4 & 0 & 0 & 0 \\
\hline
\end{tabular}


This study holds value because scientific investigation on the validity of imaging modalities for congenital pelvic abnormalities is scarce. Additionally, because this study utilized firsthand clinical data, its findings may have implication for future clinical practice. We are aware that quantifying discrepancies between imaging results and intraoperative findings inevitably involves the subjectivity of investigators. Yet, the data analysis yielded a moderately high interrater reliability. Additionally, our analysis showed that cases and controls were not significantly different from each other in terms of the majority of demographic variables. Future research should delve more deeply into this topic, while working with a larger "n," look specifically at only one CUTA such as MRKH, and look at just one imaging study, such as MRI. Most likely, this would require a multicenter study.

\section{References}

1. Chan YY, Jayaprakasan K, Zamora J, Thornton JG, Raine-Fenning N Coomarasamy A. The prevalence of congenital uterine anomalies in unsleected and high-risk populations: a systematic review. Human Reprod. 2011; 17: 761-771.

2. Creighton SM, Balen A, Breech L, Liao L. Pediatric and Adolescent Gynecology: A Problem-Based Approach. Cambridge. 2018.

3. The American College of Obstetricians and Gynecologists. Müllerian Agenesis: Diagnosis, Management, and Treatment. 2018.
4. Oppelt PG, Lermann J, Strick R, Dittrich R, Striessel P, Rettig I, et al. Malformations in a cohort of 284 women with Mayer-Rokitansky-KüsterHauser syndrome (MARKS ). Reprod Biol Endocrinol. 2012; 10: 57.

5. Hall-Craggs MA, Kirkham A, Creighton SM. Renal and urological abnormalities occurring with Mullerian anomalies. Journal of pediatric urology. 2013; 9: 27 32.

6. Berlin L. Radiologic errors, past, present and future. De Gruyter. 2014; 1 : 79-84.

7. Grimbizis GF, Di Spiezio Sardo A, Saravelos SH, Gordts S, Exacoustos C, Schoubroeck DV, et al. The Thessaloniki ESHRE/ESGE consensus on diagnosis of female genital anomalies. Gynecological surgery. 2016; 13: $1-16$.

8. Preibsch H, Rall K, Wietek BM, Brucker SY, Staebler A, Claussen CD, et al. Clinical value of magnetic resonance imaging in patients with MayerRokitansky-Küster-Hauser (MRKH) syndrome: diagnosis of associated malformations, uterine rudiments and intrauterine endometrium. Eur Radiol. 2014; 24: 1621-1627.

9. Horiguchi A. Management of male pelvic fracture urethral injuries: Review and current topics. Int J Urol. 2019; 26: 596-607. 